\title{
128. A Chromosomal Study on the 56 Cases of Cerebral Palsy
}

\author{
By Suzue Kanata,*) Tetsuji Kadotani,**) Yoko Watanabe, **) \\ Yoshio NAMBA,***) Masako NaEmURA,***) Hidetoshi Kodama,*) \\ and Setsuji KUBO*)
}

(Communicated by Sajiro Makino, M. J. A., Dec. 12, 1985)

Cerebral palsy is defined as any nonprogressive central motor deficit by the disease of brain, mainly motor center. The causal brain disease occurs at prenatal, perinatal, or postnatal period. In order to know the proportion of the chromosome abnormalities among the prenatal causes of the cerebral palsy, we made a chromosome survey on the patients who suffered from the cerebral palsy. This is a product of the direct combination-work undertaken among cytogeneticists, psychiatrists, and pediatrician. During a period from April 1982 to the end of May 1985, 56 cases with cerebral palsy, who were admitted in Hiroshima Prefectural Handicapped Children's Hospital, Wakaba Ryoikuen and in Kamo National Sanatorium, were collected for the chromosome research.

The chromosome slides were prepared following the standard blood culture procedures. The karyotype analyses were made with the application of the conventional Giemsa staining, and G- and C-banding differential staining.

Results and remarks. Table I showed the results of the clinical examinations and chromosomal findings. All patients had mental retardation; idiot, dyskinesia, difficulty or inability in walking, muscular atrophy, palsy or hemiplegia of extremities, and speech disorders. Then the above mentioned symptoms were excluded from Table I.

Fifty two cases among fifty six patients were found to have normal karyotypes, but the remaining four cases had abnormal karyotypes, being $7.1 \%$ in incidence.

They were represented by two cases of an inversion of no. 9 (inv (9) (p11q13) and $\operatorname{inv}(9)(\mathrm{p} 13 \mathrm{q} 21)$ ), one case of triplo-X (XXX), and one case of Turner's syndrome $(\mathrm{X} / \mathrm{XX} / \mathrm{Xr}(\mathrm{X}))$. The details of these cases were mentioned in the former papers (Kadotani et al. 1985 a, b; Kanata et al. 1985).

The causes of cerebral palsy fall into three main groups; prenatal, perinatal, and postnatal factors, as related to the period. Until now, the proportion of the prenatal causes has been small, and peri- and postnatal causes; asphyxia, forceps operation, pracenta praevia, inertia uteri, prolonged labor, severe jaundice, meningitis, encephalitis, and so on, have occupied the large proportion; 80-90\%. But from now, according to the advance of medicine, peri- and postnatal causes will decrease. The prenatal causes include chromosome abnormalities, hereditary diseases, intrauterine infection, and so on. The proportion of the chromosome abncrmalities among the causes of cerebral palsy has remained uncertain. More data will be needed. \footnotetext{
Hiroshima, Japan.

**) The Kadotani Medical Research Foundation. 1248, Saijohigashi, Higashihiroshima, Japan.

***) Hiroshima Prefectural Handicapped Children's Hospital, Wakaba-Ryoikuen. Hachihonmatsu-cho, Higashihiroshima, Japan.
} 
Table I. Clinical and cytogenetic findings in 56 cases

\begin{tabular}{|c|c|c|c|c|}
\hline $\begin{array}{c}\text { Case } \\
\text { no. }\end{array}$ & Age & Sex & Karyotype & Clinical features \\
\hline 1 & $8 \mathrm{y}$ & $\mathbf{F}$ & $46, \mathrm{XX}$ & $\begin{array}{l}\text { microcephaly, flat occiput, hypertelorism, mis- } \\
\text { plasia and malinplantation in teeth, hypoplastic } \\
\text { thumb, hypotonia, Lennox's syndrome }\end{array}$ \\
\hline 2 & $6 \mathrm{y}$ & M & $46, \mathrm{XY}$ & $\begin{array}{l}\text { Lecklinghausen's syndrome, dolichocephalia, } \\
\text { low-set ears, misplasia and malinplantation in } \\
\text { teeth, hypoplastic thumb, hypotonia, hypoplastic } \\
\text { external genitalia, corse hair, nevus (lt. femoral } \\
\text { region) }\end{array}$ \\
\hline 3 & $9 \mathrm{y}$. & $\mathrm{F}$ & $46, \mathrm{XX}$ & $\begin{array}{l}\text { flat occiput, hypertelorism, hypoplastic thumb, } \\
\text { club hands, club feet, calcaneous, hypotonia, } \\
\text { seizure, hypothyroidism, kidney failure }\end{array}$ \\
\hline 4 & $26 \mathrm{y}$ & M & $46, \mathrm{XY}$ & $\begin{array}{l}\text { microcephaly, flat occiput, hypertelorism, micro- } \\
\text { gnathia, misplasia and malinplantation in teeth, } \\
\text { hypoplastic external genitalia, club hands, club } \\
\text { feet, seizure }\end{array}$ \\
\hline 5 & $46 \mathrm{y}$. & $\mathrm{F}$ & $46, \mathrm{XX}$ & corse hair, flat occiput, club feet, seizure \\
\hline 6 & $11 \mathrm{y}$. & $\mathrm{F}$ & $46, \mathrm{XX}$ & flat occiput, club feet, seizure \\
\hline 7 & $2 y$. & $\mathrm{F}$ & $46, \mathrm{XX}$ & $\begin{array}{l}\text { flat occiput, strabismus, incurved index finger, } \\
\text { seizure }\end{array}$ \\
\hline 8 & $34 \mathrm{y}$. & M & $46, \mathrm{XY}$ & $\begin{array}{l}\text { microcephaly, flat occiput, strabismus, hypo- } \\
\text { plastic external genitalia, incurved fingers, } \\
\text { seizure }\end{array}$ \\
\hline 9 & $10 \mathrm{y}$ & $\mathrm{F}$ & $46, \mathrm{XX}$ & $\begin{array}{l}\text { head deformity, epicanthal fold, impaired vision, } \\
\text { seizure, hypothyroidism, asthma }\end{array}$ \\
\hline 10 & $12 \mathrm{y}$. & $\mathrm{F}$ & $46, \mathrm{XX}$ & head deformity, hypotonia, seizure \\
\hline 11 & $7 \mathrm{y}$ & M & $46, \mathrm{XY}$ & $\begin{array}{l}\text { flat occiput, ptosis of eyelid, hypoplastic ex- } \\
\text { ternal genitalia, hypotonia, hirsutism, seizure }\end{array}$ \\
\hline 12 & $44 \mathrm{y}$. & $\mathrm{F}$ & $46, \mathrm{XX}$ & $\begin{array}{l}\text { exophthalmus, strabismus, misplasia and mal- } \\
\text { inplantation in teeth }\end{array}$ \\
\hline 13 & $26 y$. & M & $46, \mathrm{XY}$ & $\begin{array}{l}\text { flat occiput, frontal bossing, hypoplastic ex- } \\
\text { ternal genitalia, incurved fingers, seizure }\end{array}$ \\
\hline 14 & $46 \mathrm{y}$ & M & $46, \mathrm{XY}$ & congenital cataracta, seizure \\
\hline 15 & $23 y$. & M & $46, \mathrm{XY}$ & hypertelorism, incurved thumb \\
\hline 16 & $13 \mathrm{y}$. & $\mathrm{F}$ & $46, \mathrm{XX}$ & $\begin{array}{l}\text { microcephaly, flat occiput, misplasia and mal- } \\
\text { inplantation in teeth, seizure }\end{array}$ \\
\hline 17 & $17 \mathrm{y}$. & $\mathrm{F}$ & $46, \mathrm{XX}$ & seizure \\
\hline 18 & $18 \mathrm{y}$. & M & $46, \mathrm{XY}$ & Sanfilippo's syndrome, saddle nose, seizure \\
\hline 19 & $13 \mathrm{y}$ & M & $46, \mathrm{XY}$ & $\begin{array}{l}\text { Sanfilippo's syndrome, saddle nose, nevus, high } \\
\text { arched palate, varus }\end{array}$ \\
\hline 20 & $6 y$. & $\mathrm{F}$ & $46, \mathrm{XX}$ & blindness, seizure \\
\hline 21 & $15 \mathrm{y}$ & $\mathrm{M}$ & $46, \mathrm{XY}$ & head deformity, strabismus, hypertonia, seizure \\
\hline 22 & $18 \mathrm{y}$. & M & $46, \mathrm{XY}$ & $\begin{array}{l}\text { ornitin transcarboamidase defect, hypertelor- } \\
\text { ism, seizure }\end{array}$ \\
\hline 23 & $48 \mathrm{y}$ & $\mathrm{F}$ & $46, \mathrm{XX}$ & $\begin{array}{l}\text { hypertelorism, saddle nose, broad nasal base, } \\
\text { upward slanted palpebral fissure }\end{array}$ \\
\hline 24 & $5 y$. & $\mathrm{F}$ & $46, \mathrm{XX}$ & strabismus, behavior disorders \\
\hline 25 & $7 \mathrm{y}$. & $\mathrm{M}$ & $46, \mathrm{XY}$ & flat occiput, strabismus, behavior disorders \\
\hline 26 & $8 \mathrm{y}$ & M & $46, \mathrm{XY}$ & $\begin{array}{l}\text { hypertelorism, strabismus, impaired vision, be- } \\
\text { havior disorders, seizure }\end{array}$ \\
\hline 27 & $2 \mathrm{y}$. & M & $46, \mathrm{XY}$ & head deformity, strabismus, seizure \\
\hline 28 & $6 \mathrm{y}$. & $\mathrm{F}$ & $47, \mathrm{XXX}$ & blindness, prominent occiput, saddle nose, cleft \\
\hline
\end{tabular}


Table I (continued)

\begin{tabular}{|c|c|c|c|c|}
\hline $\begin{array}{c}\text { Case } \\
\text { no. }\end{array}$ & Age & Sex & Karyotype & Clinical features \\
\hline & & & & $\begin{array}{l}\text { palate, misplasia and malinplantation in teeth, } \\
\text { incurved thumb, seizure }\end{array}$ \\
\hline 29 & $16 \mathrm{y}$ & $\mathrm{F}$ & $46, \mathrm{XX}$ & $\begin{array}{l}\text { hypertelorism, strabismus, saddle nose, cleft } \\
\text { palate, hypertonia, athetosis }\end{array}$ \\
\hline 30 & $32 \mathrm{y}$. & M & $46, \mathrm{XY}$ & seizure \\
\hline 31 & $26 \mathrm{y}$. & M & $46, \mathrm{XY}$ & $\begin{array}{l}\text { hypertelorism, flexion contracture of wrist, hy- } \\
\text { pertonia }\end{array}$ \\
\hline 32 & $7 \mathrm{y}$ & M & $46, \mathrm{XY}$ & $\begin{array}{l}\text { blindness, head deformity, strabismus, nystag- } \\
\text { mus, low-set ears, misplasia and malinplanta- } \\
\text { tion in teeth, seizure, murmur }\end{array}$ \\
\hline 33 & $7 \mathrm{y} \cdot$ & $\mathrm{F}$ & $46, \mathrm{XY}$ & hypertelorism, hypotonia, athetosis, seizure \\
\hline 34 & $6 \mathrm{y}$ & M & $46, \mathrm{XY}$ & $\begin{array}{l}\text { hypertelorism, upward slanted palpebral fissure, } \\
\text { strabismus, saddle nose, hypotonia, Lennox's } \\
\text { syndrome }\end{array}$ \\
\hline 35 & $7 \mathrm{y}$ & M & $46, \mathrm{XY}$ & $\begin{array}{l}\text { Lowe's syndrome, congenital cataracta, con- } \\
\text { genital glaucoma, flat occiput, upward slanted } \\
\text { palpebral fissure, hypertelorism, epicanthus, } \\
\text { misplasia and malinplantation in teeth, seizure }\end{array}$ \\
\hline 36 & $42 \mathrm{y}$ & $\mathrm{F}$ & $\begin{array}{l}46, \mathrm{XX}, \text { inv } \\
\text { (9) (p11q13) }\end{array}$ & $\begin{array}{l}\text { hypertelorism, strabismus, upward slanted pal- } \\
\text { pebral fissure, impaired vision, seizure }\end{array}$ \\
\hline 37 & $7 \mathrm{y} \cdot$ & M & $46, \mathrm{XY}$ & misplasia and malinplantation in teeth \\
\hline 38 & $1 \mathrm{y}$. & $\mathrm{F}$ & $46, \mathrm{XX}$ & $\begin{array}{l}\text { Cornelia de Langé's syndrome, narrow high } \\
\text { arched palate, impaired vision, micrognathia }\end{array}$ \\
\hline 39 & $10 \mathrm{y}$ & $\mathrm{F}$ & $46, \mathrm{XX}$ & $\begin{array}{l}\text { head deformity, hypertelorism, athetosis, sei- } \\
\text { zure }\end{array}$ \\
\hline 40 & $1 \mathrm{y}$. & $\mathbf{F}$ & $46, \mathrm{XX}$ & inverted epicanthus \\
\hline 41 & $10 \mathrm{y}$ & $\mathbf{F}$ & $46, \mathrm{XX}$ & flat occiput, seizure \\
\hline 42 & $15 \mathrm{y}$ & $\mathrm{F}$ & $46, \mathrm{XX}$ & $\begin{array}{l}\text { low nasal bridge, cleft lip, seizure, behavior dis- } \\
\text { orders }\end{array}$ \\
\hline 43 & $14 \mathrm{y}$ & M & $46, \mathrm{XY}$ & $\begin{array}{l}\text { cataracta, impaired vision, ataxia, behavior dis- } \\
\text { orders }\end{array}$ \\
\hline 44 & $12 \mathrm{y}$. & M & $46, \mathrm{XY}$ & flat occiput, club feet, club hands, seizure \\
\hline 45 & $17 \mathrm{y}$ & $\mathrm{F}$ & $46, \mathrm{XX}$ & $\begin{array}{l}\text { microcephaly, upward slanted palpebral fissure, } \\
\text { seizure }\end{array}$ \\
\hline 46 & $13 \mathrm{y}$ & M & $46, \mathrm{XY}$ & $\begin{array}{l}\text { dolichocephaly, nystagmus, equino varus, sei- } \\
\text { zure }\end{array}$ \\
\hline 47 & $18 \mathrm{y}$. & M & $46, \mathrm{XY}$ & ataxia, seizure \\
\hline 48 & $21 \mathrm{y}$ & $\mathbf{F}$ & $\begin{array}{l}45, \mathrm{X} / 46 \\
\mathrm{XX} / 46, \mathrm{Xr}(\mathrm{X})\end{array}$ & $\begin{array}{l}\text { short stature, microcephaly, hypertelorism, mis- } \\
\text { plasia and malinplantation in teeth, hypogonad- } \\
\text { ism, hypoplastic external genitalia, equino } \\
\text { varus, hypoplasia of fourth toes at both, seizure }\end{array}$ \\
\hline 49 & $16 \mathrm{y}$ & M & $46, \mathrm{XY}$ & blue sclera, impaired vision, seizure \\
\hline 50 & $34 \mathrm{y}$. & $\mathrm{F}$ & $46, \mathrm{XX}$ & $\begin{array}{l}\text { tuberous sclerosis, impaired vision, equino } \\
\text { varus, hypertonia, adenoma sebaceum }\end{array}$ \\
\hline 51 & $13 \mathrm{y}$. & M & $46, \mathrm{XY}$ & impaired vision, seizure \\
\hline 52 & $10 \mathrm{y}$ & $\mathrm{F}$ & $\begin{array}{l}46, \mathrm{XX}, \text { inv } \\
\text { (9)(p13q21) }\end{array}$ & micrognathia, seizure \\
\hline 53 & $18 \mathrm{y}$. & $\mathrm{F}$ & $46, \mathrm{XX}$ & oxycephaly, seizure \\
\hline 54 & $12 \mathrm{y}$ & $\mathrm{F}$ & $46, \mathrm{XX}$ & flat occiput, seizure \\
\hline 55 & $27 \mathrm{y}$ & M & $46, \mathrm{XY}$ & microcephaly, seizure \\
\hline 56 & $15 \mathrm{y}$ & $\mathrm{F}$ & $46, \mathrm{XX}$ & $\begin{array}{l}\text { exophthalmus, ptosis of eyelid, saddle nose, } \\
\text { seizure }\end{array}$ \\
\hline
\end{tabular}

All patients had mental retardation; idiot, dyskinesia, difficulty or inability in walking, muscular atrophy, palsy or hemiplegia of extremities, and speech disorders. 
Acknowledgements. We are cordially obliged to Emeritus Professor Sajiro Makino, M. J. A., the senior director of the Kadotani Medical Research Foundation, for improvement of this manuscript. Financial aid from the Japan Academy is gratefully acknowledged here.

\section{References}

Kadotani, T. et al. (1985a): Proc. Japan Acad., 61B, 35-36.

Kadotani, T. et al. (1985b) : ibid., 61 B, 131-133.

Kanata, S. et al. (1985): ibid., 61B, 325-328. 